\title{
A spring-embedding approach for the facility layout problem
}

\section{Castillo and T Sim}

Journal of the Operational Research Society (2010) 61, 1063. doi:10.1057/jors.2010.25

Correction to: Journal of the Operational Research Society (2004) 55, 73-81. doi: 10.1057/palgrave.jors.2601647

In Proposition 1 of Castillo and Sim (2004), we stated that the objective function of our spring-embedding model

$$
\sum_{i} \sum_{j>i} \frac{1}{2} c_{i j} d_{i j}^{2}+\sum_{i} \sum_{j>i} \max \left\{0, K_{i j}\left(r_{i}+r_{j}-d_{i j}\right)\right\}
$$

is convex. However, this statement is erroneous because $d_{i j}$ in the second term of objective function (1) is neither convex nor concave; thus, the objective function cannot be convex.

The term $d_{i j}$ is the Euclidean distance between the centers of two circles $i$ and $j$ with coordinates $\left(x_{i}, y_{i}\right)$ and $\left(x_{j}, y_{j}\right)$, respectively; that is,

$$
d_{i j}=\sqrt{\left(x_{i}-x_{j}\right)^{2}+\left(y_{i}-y_{j}\right)^{2}} \quad \forall 1 \leqslant i<j \leqslant N,
$$

with $x_{i}, y_{i}, x_{j}$, and $y_{j}$ all decision variables. It can be shown that the Hessian of $d_{i j}$ is

$$
\nabla^{2} d_{i j}=\rho\left(\mathbf{M}-\mathbf{V} \mathbf{V}^{T}\right)
$$

where

$$
\rho=\frac{1}{\sqrt{\left(x_{i}-x_{j}\right)^{2}+\left(y_{i}-y_{j}\right)^{2}}}
$$

is a positive value, $\mathbf{M}$ is the positive semidefinite matrix

$$
\mathbf{M}=\left[\begin{array}{rrrr}
1 & -1 & 0 & 0 \\
-1 & 1 & 0 & 0 \\
0 & 0 & 1 & -1 \\
0 & 0 & -1 & 1
\end{array}\right],
$$

and $\mathbf{V}$ is the vector

$$
\mathbf{V}=\left[\begin{array}{c}
\left(x_{i}-x_{j}\right) \\
-\left(x_{i}-x_{j}\right) \\
\left(y_{i}-y_{j}\right) \\
-\left(y_{i}-y_{j}\right)
\end{array}\right]
$$

The rank-one matrix $\mathbf{V} \mathbf{V}^{T}$ is positive semidefinite for any $\mathbf{V}$, which means that $-\mathbf{V} \mathbf{V}^{T}$ is negative semidefinite. Therefore, it cannot be concluded that $\nabla^{2} d_{i j}=\rho\left(\mathbf{M}-\mathbf{V} \mathbf{V}^{T}\right)$ is positive semidefinite or negative semidefinite; hence, it also cannot be stated that $d_{i j}$ is a convex function.

In closing, we note that the primary contribution of our paper is in the alternative spring-embedding formulation for the facility layout problem and the use of the augmented Lagrangian multiplier method for solving this problem. The efficiency and robustness of our proposed formulation and solution procedure are not dependent on the convexity of the objective function. 